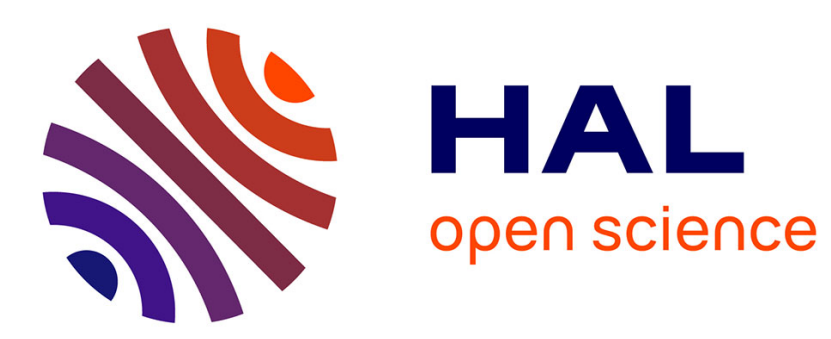

\title{
Relative Motion Threshold for Rejection in ICP Registration
}

François Pomerleau, Francis Colas, François Ferland, François Michaud

\section{To cite this version:}

François Pomerleau, Francis Colas, François Ferland, François Michaud. Relative Motion Threshold for Rejection in ICP Registration. Field and Service Robotics, Jul 2009, Cambridge, United States. 10.1007/978-3-642-13408-1_21. hal-01142575

\section{HAL Id: hal-01142575 \\ https://hal.science/hal-01142575}

Submitted on 15 Apr 2015

HAL is a multi-disciplinary open access archive for the deposit and dissemination of scientific research documents, whether they are published or not. The documents may come from teaching and research institutions in France or abroad, or from public or private research centers.
L'archive ouverte pluridisciplinaire HAL, est destinée au dépôt et à la diffusion de documents scientifiques de niveau recherche, publiés ou non, émanant des établissements d'enseignement et de recherche français ou étrangers, des laboratoires publics ou privés. 


\title{
Relative Motion Threshold for Rejection in ICP Registration
}

François Pomerleau, Francis Colas, François Ferland and François Michaud

\begin{abstract}
Simultaneous Localization and Mapping (SLAM) iteratively builds a map of the environment by putting each new observation in relation with the current map. This relation is usually done by scan matching algorithms such as Iterative Closest Point (ICP) where two sets of features are paired. However as ICP is sensitive to outliers, methods have been proposed to reject them. In this article, we present a new rejection technique called Relative Motion Threshold (RMT). In combination with multiple pairing rejection, RMT identifies outliers based on error produced by paired points instead of a distance measurement, which makes it more applicable to point-to-plane error. The rejection threshold is calculated with a simulated annealing ratio which follows the convergence rate of the algorithm. Experiments demonstrate that RMT performs better than former techniques with outliers created by dynamical obstacles. Those results were achieved without reducing convergence speed of the overall ICP algorithm.
\end{abstract}

Key words: ICP, registration, scan matching, rejection, SLAM.

\section{Introduction}

Simultaneous Localization And Mapping (SLAM) algorithms use motion and observation probabilistic models to incrementally correct positioning problems. The mechanism used to transform different observation models into the same coordinate system is called registration (also known as data association or scan matching). Proposed SLAM solutions based on Maximum Likelihood

F. Pomerleau, F. Ferland and F. Michaud

Dept. of Elec. Eng. and Computer Eng., Université de Sherbrooke, Québec, Canada

F. Pomerleau and F. Colas

Autonomous Systems Laboratory, ETH Zürich, Switzerland

e-mail: francois. [pomerleau, ferland, michaud] @usherbrooke.ca, francis.colas@mavt.

ethz.ch 
(ML) present fast capabilities to minimize global positioning errors [7], but they still need to rely on efficient and robust registration algorithms to be stable in real robotic applications.

Registration can be done using landmarks (e.g., lines, circles, arcs, corners) [1]. When applied to the registration processes, landmarks confer the advantage of accelerating calculation by summing up information. However, landmark registrations can be sensitive to unstructured environments where landmarks are difficult to detect. A second type of registration is called Normal Distribution Transform (NDT). NDT segments spatial information and works on the first and second statistical moments to reduce the computational cost [9] while avoiding to define specific landmarks. However, NDT is still very sensitive to segmentation because large spatial cells filter out relevant details, whereas small cells augment the computational cost.

Another strategy is to directly use point clouds derived from exteroceptive data. One technique to find such matches is known as Iterative Closest Point (ICP) [2]. This method pairs points of both scans by finding for each point of the first scan the nearest point in the second one. From these pairs a motion vector is estimated to cope for their misalignment. This process is iterated until convergence. ICP variants were first developed for applications involving 3D model reconstructions [4], [6], [8]. When used in SLAM by an autonomous robot, these algorithms need to be adapted in several ways: 1) they must work in real-time [3]; 2) they must be adapted to the sensors to be able to use 2D and 3D spatial information [11], and to cope with sensor fusion (range, laser reflectivity [14], color [13], etc.); 3) they must be able to deal with occlusion and partially overlapping scans that frequently arise in a dynamic environment explored by a mobile platform.

This paper addresses the issue of occlusions and partially overlapping scans by using a new adaptive rejection technique called Relative Motion Threshold (RMT). For SLAM, changes in the environment and occlusions caused by the motion of the mobile robot are sources of outliers (i.e., points with no match). Fig. 1 presents an example for which an optimal rotation and translation of the blue point cloud $\left(p_{i}\right)$ must be applied to align it with the reference red point cloud $\left(q_{j}\right)$, with $i$ and $j$ being point indexes. Even after a small displacement of the robot between $t_{1}$ and $t_{2}$, maps can largely differ due to sensor occlusion. For example, in Fig. 1(b), $50 \%$ of the blue points are outliers when compared to the red point cloud. Also, the disambiguation of points obtained from obstacle 1 and obstacle 2 must be resolved to make ICP robust to initial positioning errors and overlapping. Removing outliers from the paired points is done during the rejection step of ICP, where it minimizes misalignment errors for the determination of the motion vector between two point clouds. The rejection technique introduced in this paper uses an adaptive threshold based on simulated annealing ratio to augment the robustness of ICP against outliers.

The paper is organized as follows. Section 2 presents an overview of rejection techniques. Section 3 describes the RMT rejection technique we propose. 


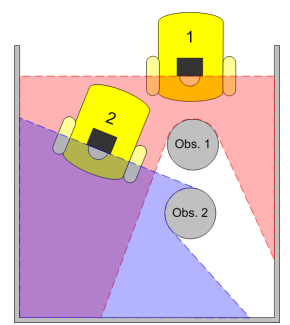

(a)

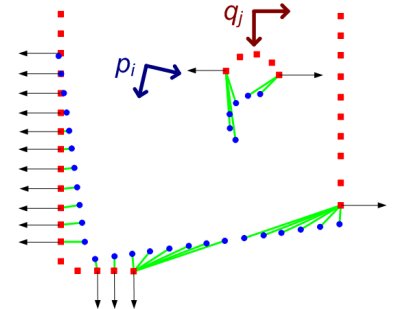

(b)

Fig. 1 Map registration example for SLAM, with misalignment caused by odometry error. The green lines represent the alignment error minimized by ICP algorithms. Obs.: Obstacle. (a) Two laser scans taken at time $t_{1}$ and $t_{2}$. (b) Point cloud $p_{i}$ (blue) taken at $t_{2}$ is represented in the coordinate system of point cloud $q_{j}$ (red) taken at $t_{1}$. Black arrows are surface orientations.

Section 4 presents experimental results that evaluate the performance of each rejection technique in terms of matching and convergence rate of ICP in simulated and real-world applications.

\section{Rejection Techniques}

Rejection techniques can be categorized as follows:

- Fix: Manual setting of the maximum distance $d$ authorized between paired points. Then, all paired points with a distance higher than $d$ are systematically rejected. This method is simple but does not adapt well to conditions that would require different thresholds.

- Zhang: Strategy based on statistical moments of the distribution of the distances between the paired points [15]. Four conditions are needed to adapt the threshold $d$ :

$$
d=\left\{\begin{array}{l}
\mu+3 \sigma, \text { if } 0 \leq \mu<\eta \\
\mu+2 \sigma, \text { if } \eta<\mu \leq 3 \eta \\
\mu+\sigma, \text { if } 3 \eta<\mu \leq 6 \eta \\
\rho, \quad \text { otherwise }
\end{array}\right.
$$

where $\rho$ is the median of the distance between paired points and $\eta$ is a distance-based parameter set by the user. This method can be adjusted to different statistical distributions of the distances but still lacks generality.

- Mean: Technique proposed in [6] which sets $d$ equal $\mu+\sigma$ for each iteration, and where $\mu$ and $\sigma$ are respectively the mean and the standard deviation of the distances between paired points. This method has no parameter 
and is flexible to different type of outliers. However it filters outliers on the assumption that the distribution of distances is Gaussian, which is not a good assumption in the case of a dynamic environment where several local minima can emerge.

- Median: An other statistical method proposed by [5] fixes $d$ to 3 times the median of the distances between paired points. The method has no parameter, but calculating a median over a huge point cloud is computationally expensive.

- Trim.: Overlapping parameter $\xi$ defined by [4] is used to reject a percentage of outliers:

$$
N_{\text {trimmed }}=\xi N_{\text {total }}
$$

where $N$ is the number of paired points. This approach is less dependent on the shape of the distribution. However, it requires to sort all paired points based on their distances at each iteration, which increases computation time. It can also misled by a large change in the overlap that may occur with a moving platform due to occlusions.

In addition to these techniques, it is also possible to reject multiple pairing to a single point [16], [3], as shown in the lower right part of Fig. 1(b) where multiple green lines connect to the same red points. Instead of authorizing all pairs, only the one with the smallest distance is kept. This criterion has been shown to improve the performance of all standard rejection techniques, and thus all results presented here use this additional criterion.

\section{Relative Motion Threshold Technique for Rejection}

Existing rejection techniques rely mostly on the Euclidean distance between paired points. While this distance has a direct impact on point-to-point error metric, ICP implementations commonly use a point-to-plane error metric to pair the points because of its faster convergence speed. This point-toplane error metric ${ }^{1}$ assumes that there is a local surface orientation vector estimated for each point $q_{j}$ and projects the Euclidean distance between $p_{i}$ and $q_{j}$ on this vector. The point paired to $q_{j}$ then minimizes this error and not the Euclidean distance (see Fig. 1(b)). We introduce a new, more general, rejection technique called Relative Motion Threshold (RMT). RMT is an adaptive rejection technique that progressively identifies outliers that create most of the error during the process of ICP. Adaptation is based directly on the error created by paired points instead of the Euclidean distance between those points in accordance to the matching process. We propose to reject the outliers with a maximum authorized error $e_{t}$ at iteration $t$, evaluated by:

\footnotetext{
${ }^{1}$ In the remaining of the text, the term error will refer to the point-to-plane error metric when there is no ambiguity.
} 


$$
e_{t}=\left\{\begin{array}{cc}
\lambda e_{t-1}, & \text { if } \lambda<1 \\
e_{t-1}, & \text { otherwise }
\end{array}\right.
$$

with $\lambda$ being a simulated annealing ratio defined by:

$$
\lambda=\frac{\left\|T_{t-1}\right\|}{\left\|T_{t-2}\right\|}
$$

This ratio uses past motion information to determine if the point cloud is converging to a local minima, where $\|T\|$ is the Euclidean norm of the translation vector $T$ which minimize the alignment error of $p_{i}$ at iteration $t$. The translation vector $T$ and the rotational vector $\Omega$ are calculated during the Error and Minimization step at the end of each iteration and are used to move $p_{i}$ toward $q_{i}$. A ratio $\lambda$ smaller than 1 means that the position of $p_{i}$ is stabilizing. All points with a translation error larger than $e_{t}+\epsilon$ are identified as outliers and rejected during the iteration $t$. If the ratio $\lambda$ is larger than 1 , the motion of point cloud $p_{i}$ is accelerating toward $q_{i}$ due to new appearing constraints. The maximum authorized error $e_{t}$ is then kept stable until the point cloud starts to converge again.

The minimum error $\epsilon$ is the only parameter needed for our rejection technique. It represents noises from sensor readings. A simple way to evaluate this parameter is to take two scans of a static environment and look at the distribution of translation errors created by the error metric used. This translation error should be centered on zero and can be estimated by a Gaussian distribution. The parameter $\epsilon$ can be estimated using the standard deviation of this distribution, making the parameter sensor-dependent instead of situation-dependent.

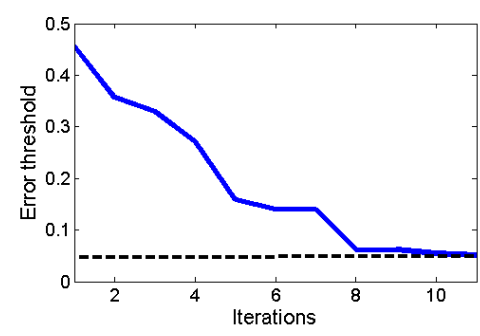

(a)

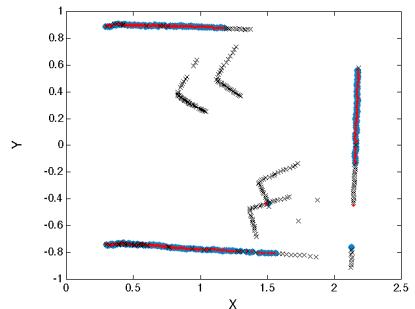

(b)

Fig. 2 (a) A generic example of maximal authorized error based on RMT in function of iterations. Dashed line represents minimum error $\epsilon$. (b) Final position of the registered point clouds used in the generic example.

Fig. 2(a) presents an example of the relative motion threshold in function of iterations. During the two first iterations, only the rejection of multiple pairings is active to initialize a value for $T_{t-1}$ and $T_{t-2}$. Then, $e_{2}$ is initialized 
to the maximum Euclidean norm of translation error at iteration 2 . The simulated annealing ratio reduces this error until the point cloud $p_{i}$ temporarily stops converging. The ratio $\lambda$ is higher than 1 between iteration 6 and 7 , forcing a constant error threshold until the point cloud $p_{i}$ start converging again. The final threshold error is reduced until iteration 11 where it equals the minimum error $\epsilon$ represented by the dashed line. Fig. 2(b) shows the two point clouds used in this example at iteration 11. The point clouds were taken in a room where two boxes were moved in different directions. The thick points in light blue represent $p_{i}$ which converges to the right position even with outliers caused by dynamic obstacles (i.e., boxes). Small black cross represent outliers detected by RMT.

\section{Experimental Results}

Experiments were conducted in simulation and real settings using the following ICP algorithm consisting in five steps [12]:

1. Selection reduces the number of points in $p_{i}$ by selecting a representative subset of points $p_{s}$, where $s<i$. This step is a compromise between computation speed and robustness. Even if using a smaller number of points results in faster computation time, the result may diverge if not enough points are used, or if the selection process filters out necessary constraints.

2. Matching pairs each point of $p_{s}$ in the point cloud $q_{j}$. This corresponds to a closest point search problem. One data structure often used to solve this problem is the $\mathrm{k}-\mathrm{d}$ tree. It is a data structure that partitions the space into $k$ dimensions, with the property of accelerating the nearest neighbor search. Recently, utilization of the approximate k-d tree [8] has shown to give faster results without altering ICP precision.

3. Weighting improves or reduces impacts of pairing point on the error matrix by using criteria such as distance, normal compatibilities and scanner noise. However, results suggest that weighting is data-dependent and does not increase convergence rate significantly [12].

4. Rejection uses techniques described in Section 2 and 3.

5. Error and minimization use all the remaining matched points to evaluate the misalignment error and a create a motion vector $m=[T, \Omega]^{\prime}$ minimizing this error where $T$ is the translation components and $\Omega$ the orientation components. This motion vector is applied to the point cloud $p_{i}$. Pointto-plane error function is shown to have a faster convergence rate than point-to-point error [12].

Steps 2 to 5 are repeated until any of the ending condition is reached. Several ending conditions have been proposed, e.g. number of iterations, error, relative motion between two iterations [4], [16], stabilization of mean 
and standard deviation of the distances between paired points, number of registered pairs [6]. Our complete ICP algorithm uses all of those.

\subsection{Evaluation method}

To test the RMT rejection technique while dealing with outliers or occlusion caused by moving objects, we enriched the test protocol described in [10]. More specifically, we recorded data taken by a SICK LMS 200 laser range finder in a U-shaped room. Without moving the sensor, we added or moved boxes in its field of view. This way we generated 10 pairs of different scans with an overlapping ratio around $75 \%$. For each trial, one of the two scans was transformed with a rotation and a translation vector drawn randomly according to a Gaussian distribution in order to fit the uncertainty of the localization of standard SLAM techniques. The standard deviations were $0.15 \mathrm{~m}$ for each translation component and $0.15 \mathrm{rad}$ for the angle. Fig. 3(a) shows an example of two scans as well as the distribution of displacement of the second scan. We can see that in the second scan, one of the boxes moved while the other was removed. As the sensor is fixed between the scans, the result of the registration algorithm is exactly the inverse of this transformation. Fig. 3(b) shows the results for each rejection techniques. Curves represent the mean of the XY alignment error of $p_{i}$ over 4000 trials. For rejection techniques that require the setting of parameters, optimal values were derived by sampling the parameter space and computing the percentage of good registration over a training set. The final performances were evaluated using the remaining configurations. Theses optimal parameters are presented in Table 1. RMT provides a large improvement over the other rejection techniques, since other methods tend to wrongly categorize points as outliers and converge towards local minima.

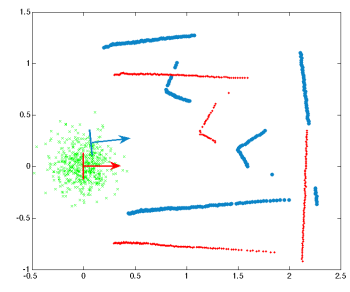

(a)

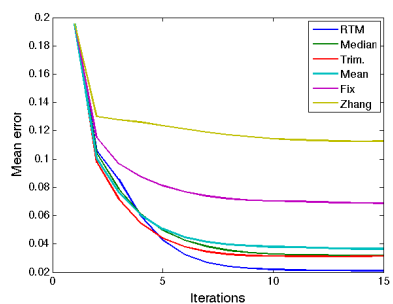

(b)

Fig. 3 (a) Two scans to be matched. The first scan is in red, whereas the second scan, after translation and rotation, is in thick blue. The green crosses show the distribution of displacement error used in our test with a standard deviation of 0.15 on $\mathrm{x}$ - and y-axis. (b) Comparison of the performance of several rejection methods in function of iterations. The performance is measured by the mean position error in respect to ground truth. 


\begin{tabular}{|l||l|l|l|l|l|l|}
\hline & RMT & Median & Trim. & Mean & Fix & Zhang \\
\hline \hline Parameter & $\epsilon=0.05$ & none & $\xi=76 \%$ & none & $d=0.3 \mathrm{~m}$ & $\eta=0.02 \mathrm{~m}$ \\
\hline
\end{tabular}

Table 1 Parameters used for each rejection techniques during comparison test.

In terms of speed, ICP is an iterative algorithm known to converge in a small number of iterations. RMT rejection method does not impair the convergence rate of the matching algorithm. The mean and covariance on the number of iterations for the Median and RMT are respectively $(\mu=9.5$, $\sigma=2.4)$ and $(\mu=9.9, \sigma=1.9)$. Those results were obtained while keeping registration converging to the right value for 4000 trials. No significant difference were observed between all rejection techniques tested.

Looking at how rejection techniques perform with shifting initial positions, Table 2 presents the correct registration computed for 4000 trials of each rejection technique for various standard deviations on the initial error. RMT rejection technique performs better than the others for all conditions tested by having the lowest residual error. Moreover, a rise in the initial position variance decreases the performance for every methods, as expected. For this setup, it means that uncertainty on the position of the robot should be kept under $15 \mathrm{~cm}$ before applying ICP to achieve good registration. However, RMT rejection method is more robust than other techniques as performance loss occurs at a higher variance while being less computationally expensive than median technique.

\begin{tabular}{|c||c|c|c|c|c|c|}
\hline Std & RMT & Median & Trim. & Mean & Fix & Zhang \\
\hline \hline 0.05 & 0.001 & 0.005 & 0.013 & 0.009 & 0.027 & 0.021 \\
\hline 0.10 & 0.004 & 0.016 & 0.015 & 0.013 & 0.029 & 0.056 \\
\hline 0.15 & 0.021 & 0.031 & 0.032 & 0.039 & 0.055 & 0.107 \\
\hline 0.20 & 0.034 & 0.051 & 0.060 & 0.059 & 0.077 & 0.132 \\
\hline 0.30 & 0.135 & 0.145 & 0.189 & 0.203 & 0.261 & 0.282 \\
\hline 0.40 & 0.272 & 0.294 & 0.356 & 0.362 & 0.412 & 0.423 \\
\hline
\end{tabular}

Table 2 Robustness of the rejection techniques with respect to initial error. The performance is measured in term of the mean final of $\mathrm{XY}$ alignment error of point clouds (in meter).

\subsection{Real-world application}

The last section described experiments with outliers mainly due to dynamical obstacles. Another main source of outliers can be created by low overlapping percentage of scans. The Canadian Space Agency (CSA) uses a rotating laser range finder installed on a robot to test Mars exploration algorithms which is showed in Fig. 4 (a). The robot typically moves few meters on a simulated Martian terrain, takes a 3D scan and decides where to go next. In this kind 
of application, overlapping between scans can vary between 50\% and $90 \%$ and few 3D features are available, making the registration very sensible to outliers. The RMT was used on 3D point clouds extracted within this context of application. Fig. 4 presents the result of one registration with a distance of $15 \mathrm{~m}$ between the two scans. The grayscale surface correspond to the section used for the registration. The maximum height of the surface is about $1 \mathrm{~m}$. Points on both side of the surface represent outliers removed during the registration. This demonstrates that the RMT can also deal with outliers created by low overlapping scans. Moreover, the algorithm is currently used by the Space Technologies Research Program of the CSA for complete mapping of the experimental Martian terrain.

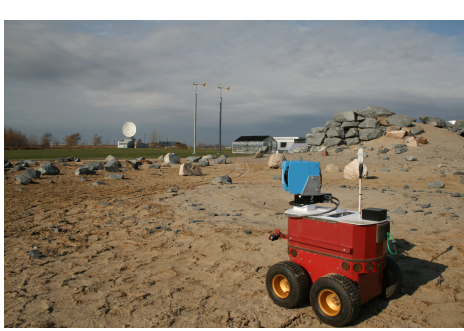

(a)

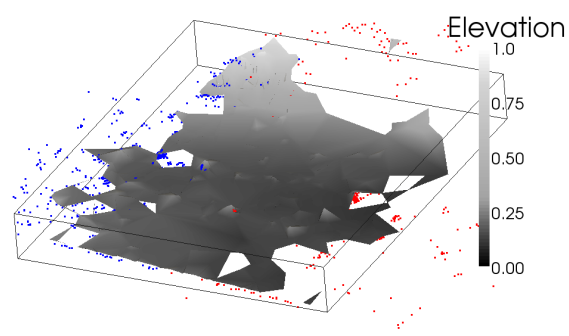

(b)

Fig. 4 (a) Robot and environment of the Canadian Space Agency for the Mars exploration project. (b) RMT applied to scans with low overlapping. In grayscale, the surface recovered from match points. In red and blue, the outliers of each scan.

\section{Conclusion and Future Work}

This paper presents a novel rejection technique called RMT in the context of ICP registration applied to SLAM in mobile robotics. Results show promising performance, making RMT a very interesting alternative to other rejection techniques. In particular, RMT allows better registration with point clouds containing dynamical obstacles. RMT also demonstrates its applicability in a Mars exploration context with low overlapping percentages. It also gives good results for identifying dynamical obstacles. In future work, we plan to characterize the stability of the approach in a complete SLAM algorithm, and to further extend the range of initial error that ICP can resolve.

\section{Acknowledgments}

The authors gratefully acknowledge the contribution of the Natural Sciences and Engineering Research Council of Canada (CRSNG), the Canada Re- 
search Chairs (CRC) and the Fondation UdeS for their financial support. We extend our thanks to David Gingras for his work realized under the Space Technologies Research Program at CSA. This work was also partially supported by Robots@home STREP EU Project IST-6-045350.

\section{References}

1. M. Altermatt, A. Martinelli, N. Tomatis, and R. Siegwart. Slam with corner features based on a relative map. Intelligent Robots and Systems (IROS). Proceedings of the IEEE/RSJ International Conference on, 2:1053-1058, Sept., Oct. 2004.

2. P. Besl and H. McKay. A method for registration of 3-d shapes. Pattern Analysis and Machine Intelligence, IEEE Transactions on, 14(2):239-256, Feb 1992.

3. A. Censi. An icp variant using a point-to-line metric. Robotics and Automation (ICRA). IEEE International Conference on, pages 19-25, May 2008.

4. D. Chetverikov, D. Svirko, D. Stepanov, and P. Krsek. The trimmed iterative closest point algorithm. Pattern Recognition. Proceedings. 16th International Conference on, 3:545-548, 2002.

5. J. Diebel, K. Reutersward, S. Thrun, J. Davis, and R. Gupta. An icp variant using a point-to-line metric. Intelligent Robots and Systems (IROS) IEEE/RSJ International Conference on, pages 3436-3443, Sept., Oct. 2004.

6. S. Druon, M. Aldon, and A. Crosnier. Color constrained icp for registration of large unstructured 3d color data sets. Information Acquisition. IEEE International Conference on, pages 249-255, Aug. 2006.

7. G. Grisetti, S. Grzonka, C. Stachniss, P. Pfaff, and W. Burgard. Efficient estimation of accurate maximum likelihood maps in 3D. Proceedings of IEEE/RSJ International Conference on Intelligent Robots and Systems, pages 3472-3478, October 2007.

8. N. Ho and R. Jarvis. Large scale $3 \mathrm{~d}$ environmental modelling for stereoscopic walkthrough visualisation. 3DTV Conference, pages 1-4, May 2007.

9. B. Huhle, M. Magnusson, W. Strasser, and A. Lilienthal. Registration of colored 3d point clouds with a kernel-based extension to the normal distributions transform. Robotics and Automation (ICRA). IEEE International Conference on, pages 40254030, May 2008.

10. J. Minguez. Metric-based scan matching algorithms for mobile robot displacement estimation. In In Int. Conf. on Robotics and Automation, 2005.

11. A. Nuchter, K. Lingemann, J. Hertzberg, and H. Surmann. 6d slam with approximate data association. Advanced Robotics, 2005 (ICAR). Proceedingsof the 12th International Conference on, pages 242-249, July 2005.

12. S. Rusinkiewicz and M. Levoy. Efficient variants of the icp algorithm. Proceeding on 3DIM, pages 145-152, 2001.

13. M. Strand, F. Erb, and R. Dillmann. Range image registration using an octree based matching strategy. Mechatronics and Automation (ICMA). International Conference on, pages 1622-1627, Aug. 2007.

14. H. Yoshitaka, K. Hirohiko, O. Akihisa, and Y. Shin'ichi. Mobile robot localization and mapping by scan matching using laser reflection intensity of the sokuiki sensor. IEEE Industrial Electronics (IECON). 32nd Annual Conference on, pages 3018-3023, Nov. 2006.

15. Z. Zhang. Iterative point matching for registration of free-form curves and surfaces. International Journal of Computer Vision, 13(2), 1994.

16. T. Zinsser, J. Schmidt, and H. Niemann. A refined icp algorithm for robust 3-d correspondence estimation. Image Processing (ICIP). Proceedings. 2003 International Conference on, 2:II-695-8 vol.3, Sept. 2003. 